\title{
Photoerasable Organic Field-Effect Transistor Memory Based on a One-Step Solution-Processed Hybrid Floating Gate Layer
}

Qingyan Li, ${ }^{\dagger}$ Tengteng Li, ${ }^{\dagger}$ Yating Zhang, ${ }^{*}$ Zhiliang Chen, Yifan Li, Lufan Jin, Hongliang Zhao, Jie Li, Jianquan Yao

Key Laboratory of Opto-Electronics Information Technology, Ministry of Education, School of Precision Instruments and Opto-Electronics Engineering, Tianjin University, Tianjin 300072, China

These authors contributed equally 

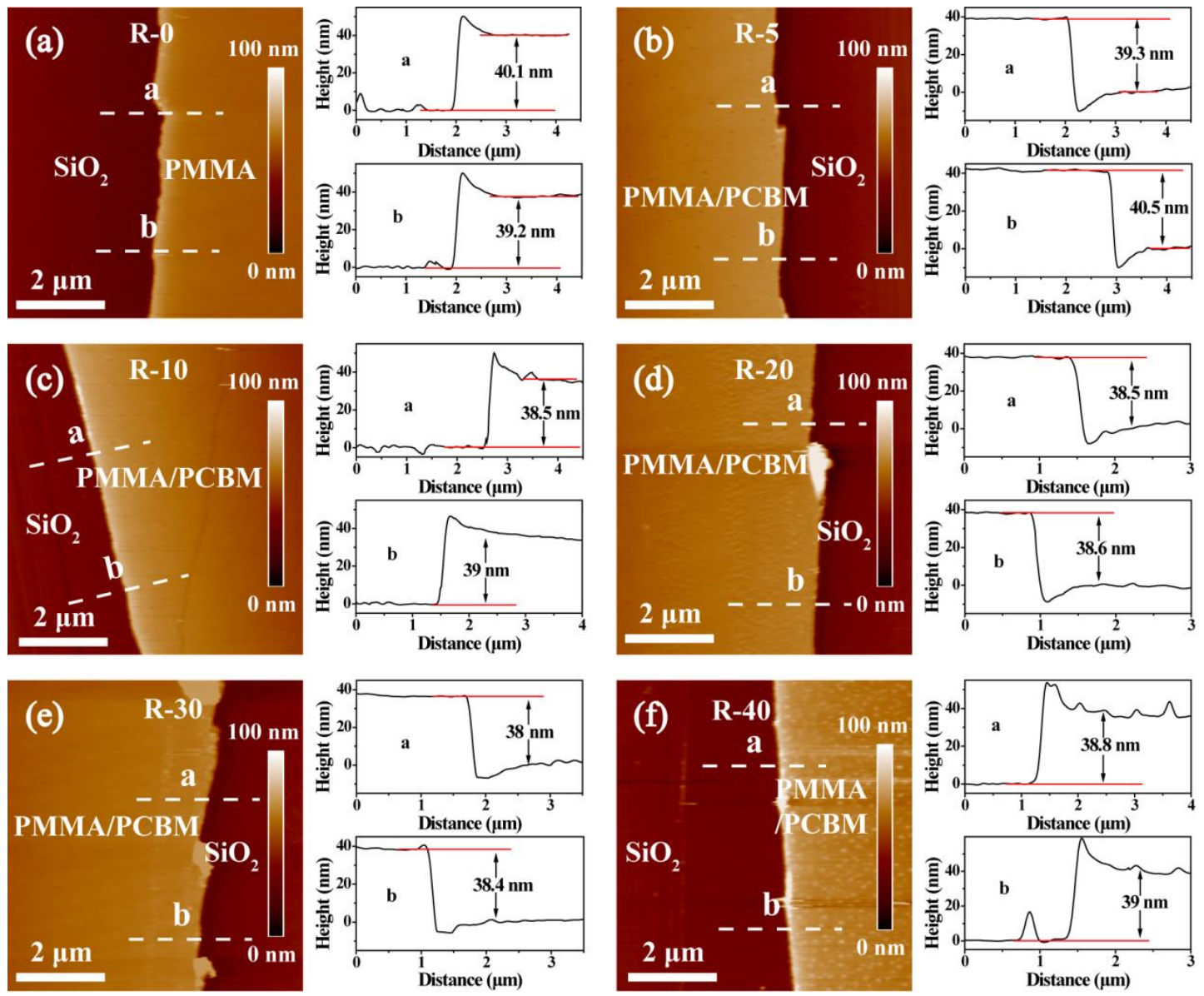

Figure S1. AFM height images of (a) R-0, (b) R-5, (c) R-10, (d) R-20, (e) R-30, and (f) R-40 films on the bare $\mathrm{SiO}_{2}$, corresponding cross-sectional profile of the PCBM/PMMA composite layers.

We measured the thickness of R-0, R-5, R-10, R-20, R-30, and R-40 films on the bare $\mathrm{SiO}_{2}$ by AFM. The films were slit by a needle to facilitate of height measurement. As shown in Figure S1, the thicknesses of the PCBM/PMMA composite layers with various blending ratios were about $38 \sim 40.5 \mathrm{~nm}$. The thicknesses of the composite films with various blending ratios were not much different, eliminating the effect of thickness on memory windows. 

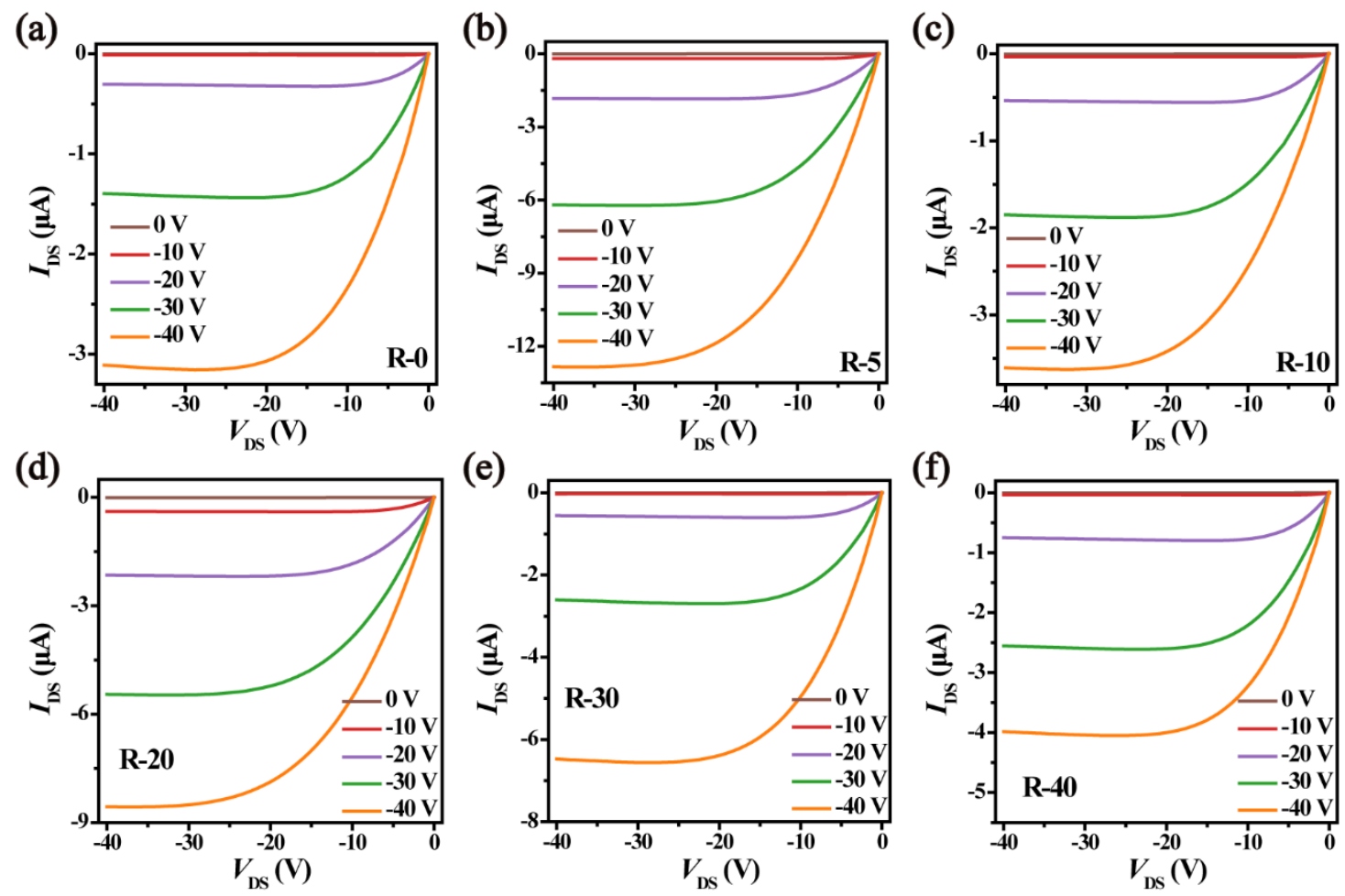

Figure S2. Output characteristics of (a) R-0, (b) R-5, (c) R-10, (d) R-20, (e) R-30, and (f) R-40 memory devices. 

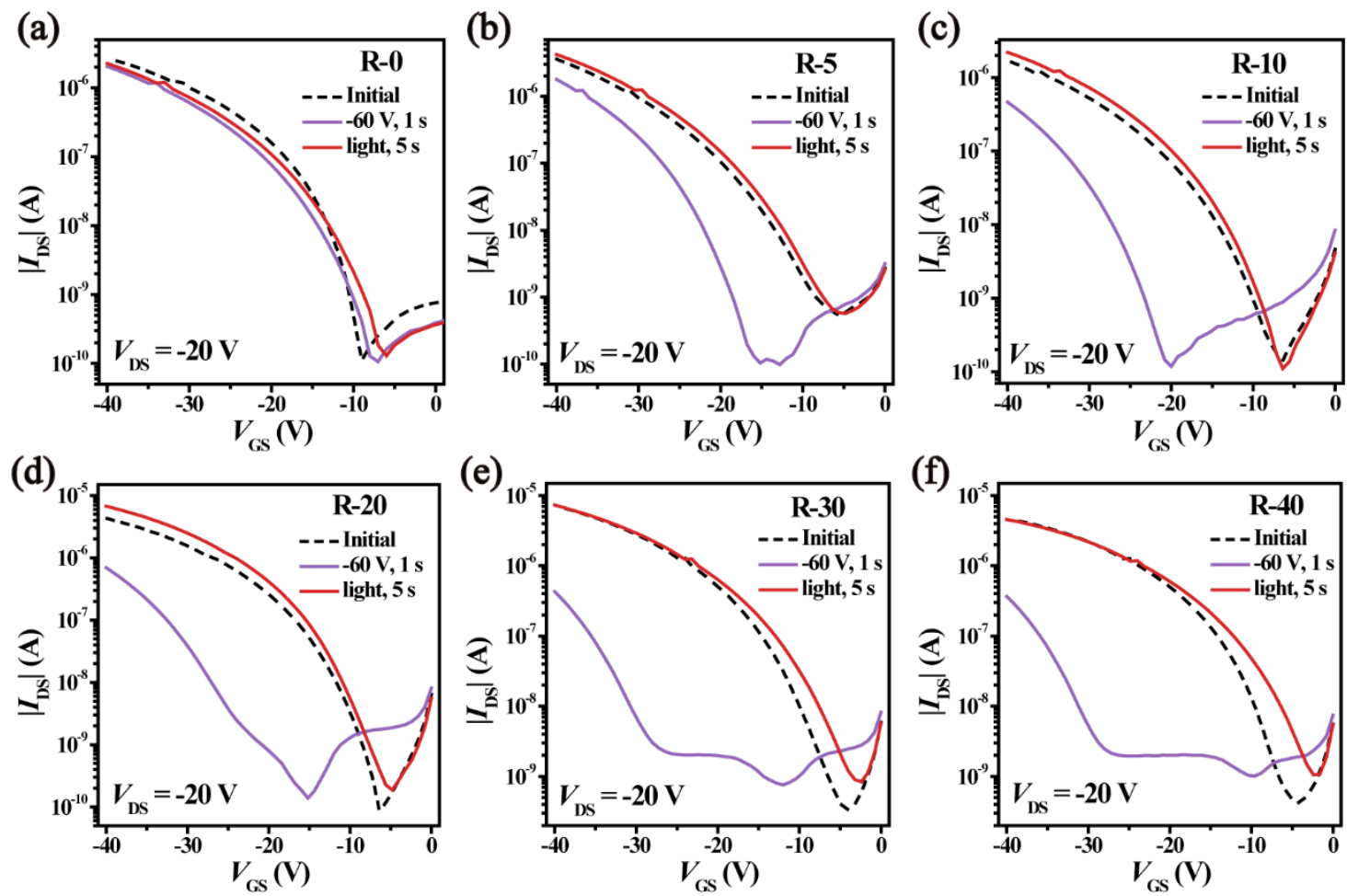

Figure S3. Memory characteristics ( $\log \left|I_{\mathrm{DS}}\right|$ versus $V_{\mathrm{GS}}$ curves) of (a) R-0, (b) R-5, (c) R-10,

(d) R-20, (e) R-30, and (f) R-40 memory devices at initial states, programmed states and erased states. 


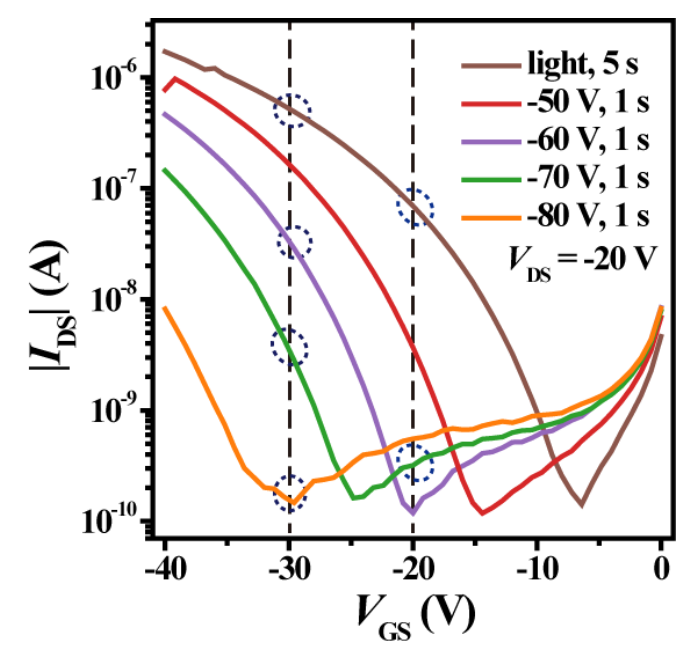

Figure S4. Transfer characteristics ( $\log \left|I_{\mathrm{DS}}\right|$ versus $V_{\mathrm{G}}$ curves) of R-10 memory device after electronic programming operations with different programming voltages and light erasing operations. 


\section{Threshold voltage calculation:}

The threshold voltage $\left(V_{\mathrm{th}}\right)$ can be obtained from the intersection of the extension line and the abscissa by extrapolating a liner plot of $\left|I_{\mathrm{DS}}\right|^{1 / 2}$ versus $V_{\mathrm{GS}}$ to $\left|I_{\mathrm{DS}}\right|^{1 / 2}$ equal to 0 according to the following equation: ${ }^{1-3}$

$$
I_{\mathrm{DS}}=\mu \frac{W}{2 L} C_{i}\left(V_{\mathrm{GS}}-V_{\mathrm{th}}\right)^{2}
$$

where $W, L, V_{\mathrm{GS}}, I_{\mathrm{DS}}$ and $C_{\mathrm{i}}$ are the channel width, channel length, gate-source voltage, drain-source current and the capacitance per unit area of the gate insulator, respectively.

Table S1. Threshold voltages of initial states, electrical programmed states $(-60 \mathrm{~V}, 1 \mathrm{~s})$, photo-erased states $\left(532 \mathrm{~nm}, 3 \mathrm{~mW} \mathrm{~cm}^{-2}, 5 \mathrm{~s}\right)$, and corresponding memory windows of R-0, R-5, R-10, R-20, R-30, and R-40 memory devices.

\begin{tabular}{|c|c|c|c|c|}
\hline $\begin{array}{l}\text { Memory } \\
\text { devices }\end{array}$ & $\begin{array}{c}V_{\text {th }} \text { (initial) } \\
\text { [V] }\end{array}$ & $\begin{array}{c}V_{\text {th }}(-60 \mathrm{~V}) \\
{[\mathrm{V}]}\end{array}$ & $\begin{array}{c}V_{\text {th }} \text { (light) } \\
\text { [V] }\end{array}$ & $\begin{array}{l}\Delta V_{\text {th }} \\
{[\mathrm{V}]}\end{array}$ \\
\hline R-5 & -16.7 & -24.4 & -15.4 & 9.0 \\
\hline R-10 & -17.1 & -27.9 & -15.9 & 12.0 \\
\hline R-20 & -13.9 & -28.4 & -13.8 & 14.6 \\
\hline R-30 & -14.1 & -31.3 & -13.0 & 18.3 \\
\hline $\mathrm{R}-40$ & -11.1 & -31.5 & -9.4 & 22.1 \\
\hline
\end{tabular}


Table S2. Threshold voltages of R-10 memory device after electronic programming operations with different programming voltages.

\begin{tabular}{ccccccc}
\hline $\begin{array}{c}\text { Memory } \\
\text { device }\end{array}$ & $V_{\text {th }}(-30 \mathrm{~V})$ & $V_{\text {th }}(-40 \mathrm{~V})$ & $V_{\text {th }}(-50 \mathrm{~V})$ & $V_{\text {th }}(-60 \mathrm{~V})$ & $V_{\text {th }}(-70 \mathrm{~V})$ & $V_{\text {th }}(-80 \mathrm{~V})$ \\
\hline R-10 & {$[\mathrm{V}]$} & {$[\mathrm{V}]$} & {$[\mathrm{V}]$} & {$[\mathrm{V}]$} & {$[\mathrm{V}]$} & {$[\mathrm{V}]$} \\
\hline
\end{tabular}

Table S3. Threshold voltages of R-10 memory device after the electronic programming operations with different programming times.

\begin{tabular}{cccccc}
\hline Memory & $V_{\text {th }}(1 \mathrm{~ms})$ & $V_{\text {th }}(10 \mathrm{~ms})$ & $V_{\text {th }}(100 \mathrm{~ms})$ & $V_{\text {th }}(500 \mathrm{~ms})$ & $V_{\text {th }}(1 \mathrm{~s})$ \\
device & {$[\mathrm{V}]$} & {$[\mathrm{V}]$} & {$[\mathrm{V}]$} & {$[\mathrm{V}]$} & {$[\mathrm{V}]$} \\
\hline R-10 & -23.4 & -24.8 & -25.6 & -28.1 & -31.4 \\
\hline
\end{tabular}


Table S4. Threshold voltages of R-10 memory device after photo-erasing operations with different light intensities under $532 \mathrm{~nm}$ and $405 \mathrm{~nm}$ light pulse illumination with pulse width of $5 \mathrm{~s}$, respectively.

\begin{tabular}{ccccc}
\hline light & $0.5 \mathrm{~mW} / \mathrm{cm}^{2}$ & $1 \mathrm{~mW} / \mathrm{cm}^{2}$ & $2 \mathrm{~mW} / \mathrm{cm}^{2}$ & $3 \mathrm{~mW} / \mathrm{cm}^{2}$ \\
\hline $532 \mathrm{~nm}$ & -21.4 & -19 & -17.4 & -15.9 \\
$405 \mathrm{~nm}$ & -21.5 & -19.4 & -17.6 & -15.6 \\
\hline
\end{tabular}

Table S5. Threshold voltages of R-10 memory device after photo-erasing operations under $532 \mathrm{~nm}$ and $405 \mathrm{~nm}$ light pulse illumination with different pulse width at an intensity of 3 $\mathrm{mw} / \mathrm{cm}^{2}$, respectively.

\begin{tabular}{ccccc}
\hline light & $500 \mathrm{~ms}$ & $1 \mathrm{~s}$ & $3 \mathrm{~s}$ & $5 \mathrm{~s}$ \\
\hline $532 \mathrm{~nm}$ & -21.4 & -20.4 & -17.6 & -15.9 \\
$405 \mathrm{~nm}$ & -22.1 & -20.7 & -18.1 & -15.6 \\
\hline
\end{tabular}




\section{REFERENCES}

(1) Zhuang, J.; Lo, W. S.; Zhou, L.; Sun, Q. J.; Chan, C. F.; Zhou, Y.; Han, S. T.; Yan, Y.; Wong, W. T.; Wong, K. L.; et al. Photo-reactive charge trapping memory based on lanthanide complex. Sci. Rep. 2015, 5, 14998.

(2) Li, Q.; Li, T.; Zhang, Y.; Yu, Y.; Chen, Z.; Jin, L.; Li, Y.; Yang, Y.; Zhao, H.; Li, J.; et al. Nonvolatile photoelectric memory with $\mathrm{CsPbBr}_{3}$ quantum dots embedded in poly(methyl methacrylate) as charge trapping layer. Org. Electron. 2020, 77.

(3) Han, S.-T.; Zhou, Y.; Zhou, L.; Yan, Y.; Huang, L.-B.; Wu, W.; Roy, V. A. L. $\mathrm{CdSe} / \mathrm{ZnS}$ core-shell quantum dots charge trapping layer for flexible photonic memory. $J$. Mater. Chem. C 2015, 3, 3173-3180. 